\title{
Diagrammatic Approach for Investigating Two Dimensional Elastic Collisions in Momentum Space II: Special Relativity
}

\author{
Akihiro Ogura \\ Laboratory of Physics, Nihon University, Matsudo, Japan \\ Email: ogura.akihiro@nihon-u.ac.jp
}

How to cite this paper: Ogura, A. (2018) Diagrammatic Approach for Investigating Two Dimensional Elastic Collisions in Momentum Space II: Special Relativity. World Journal of Mechanics, 8, 353-361. https://doi.org/10.4236/wjm.2018.89026

Received: August 2, 2018

Accepted: August 28, 2018

Published: August 31, 2018

Copyright $\odot 2018$ by author and Scientific Research Publishing Inc. This work is licensed under the Creative Commons Attribution International License (CC BY 4.0).

http://creativecommons.org/licenses/by/4.0/

\begin{abstract}
The diagrammatic approach to the collision problems in Newtonian mechanics is useful. We show in this article that the same technique can be applied to the case of the special relativity. The two circles play an important role in Newtonian mechanics, while in the special relativity, we need one circle and one ellipse. The circle shows the collision in the center-of-mass system. And the ellipse shows the collision in the laboratory system. These two figures give all information on two dimensional elastic collisions in the special relativity.
\end{abstract}

\section{Keywords}

Two Dimensional Elastic Collision, Momentum Space, Laboratory System, Center-of-Mass System, Special Relativity

\section{Introduction}

Collisions of the interacting particles have fundamental importance in physics. We often use the accelerated particles to investigate the substances. Cosmic ray which is often accelerated up to almost the speed of light collides with other particles in the air. For those particles which have high energy, special relativity has to be considered to investigate the collisions.

Diagrammatic technique gives the powerful tool to investigate the collision in Newtonian mechanics [1] [2] [3]. In this article, we apply it to the relativistic collision problems [4] [5]. The two circles played an important role in Newtonian mechanics, while in the special relativity one circle and one ellipse play a crucial role. When the speed of the projectile tends to small compared to the speed of light, the ellipse becomes a circle and the Newtonian case recover in 
this limit [5].

This paper is organized in the following way. In Section 2, we recall two dimensional elastic collisions with equations. In Section 3, we show the diagrammatic approach for two dimensional elastic collision in order. First, we draw a circle for the center-of-mass system. Then we add to draw an ellipse to obtain the momentum after the collision in the laboratory system. In Section 4, we investigate the special case in which the two particles are identical. We also compare the cases that the projectile has different speed and we find that the low speed limit recovers the Newtonian case [3] [5]. Section 5 is devoted to a conclusion.

\section{Elastic Collision between Two Particles in Two Dimensions}

Let us take a look the two dimensional elastic collision for later use. Figure 1 shows the collisions from the point of view in the laboratory and center-of-mass system and also show the notation which we use in this article. The projectile A has mass $m_{A}$ and the velocity $\boldsymbol{v}_{A}$ and the target $\mathrm{B}$ has mass $m_{B}$ and the velocity $v_{B}$ before the collision. These quantities are known parameters or initial conditions in the laboratory system. The velocities after the collision are distinguished by the primes. And the asterisk is attached to the parameter in the center-of-mass system. In this article, we restrict ourselves that the target particle is at rest $\boldsymbol{v}_{B}=\mathbf{0}$ in the laboratory system before the collision.

The relation between the laboratory and center-of-mass systems is governed by the Lorentz transformation [6]. Let $\beta=V / c$ be the relative velocity between two systems and is given by

$$
\beta=\frac{p_{A}}{E_{A} / c+m_{B} c},
$$

where $c$ is the speed of light. The momentum $p_{A}$ is defined by its velocity $v_{A}$ as $p_{A}=m_{A} v_{A} / \sqrt{1-\left(v_{A} / c\right)^{2}}$ and the energy is given by $E_{A} / c=\sqrt{p_{A}^{2}+\left(m_{A} c\right)^{2}}$. The $\gamma$-factor is obtained by

$$
\gamma=\frac{1}{\sqrt{1-\beta^{2}}}=\frac{E_{A} / c+m_{B} c}{\sqrt{\left(m_{A} c\right)^{2}+\left(m_{B} c\right)^{2}+2 E_{A} m_{B}}} .
$$
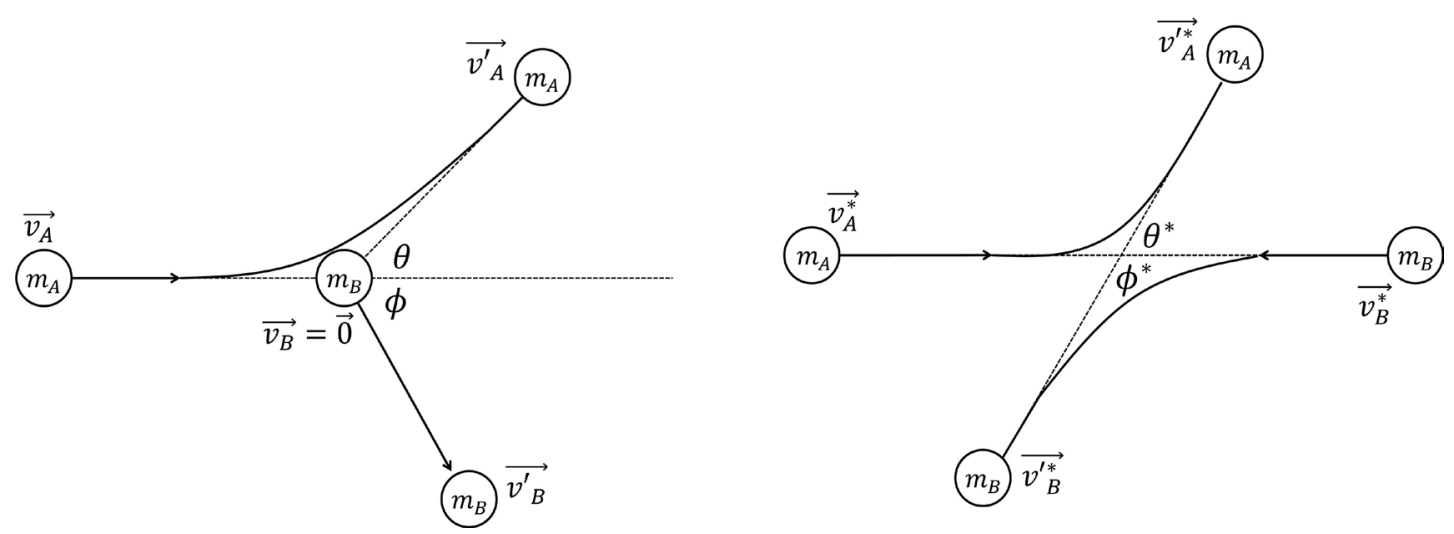

Figure 1. Left: Collisions in the laboratory system. Right: Collisions in the center-of-mass system. 
From the Lorentz transformation, the momentum of the incident particles in the center-of-mass system is given by

$$
p^{*}=p_{A}^{*}=p_{B}^{*}=\frac{p_{A} m_{B} c}{\sqrt{\left(m_{A} c\right)^{2}+\left(m_{B} c\right)^{2}+2 E_{A} m_{B}}},
$$

where note that the momenta in the center-of-mass system are the same in magnitude after the collision: $p^{*}=p_{A}^{\prime *}=p_{B}^{\prime *}$.

In the same way as the Newtonian mechanics [3], let $\boldsymbol{n}^{*}=\left(\cos \theta^{*}, \sin \theta^{*}\right)$ be the scattering angle of the projectile after the collision in the center-of-mass system. Since the angle $\theta^{*}$ is not determined by the conservations of energy and momentum, we fix it according to the collision problems. Let $\boldsymbol{p}_{A}^{\prime}=\left(p_{A x}^{\prime}, p_{A y}^{\prime}\right)$ be the $x, y$-components of the momentum of the projectile in the laboratory system after the collision. The Lorentz transformation gives the relation between the laboratory system and center-of-mass system as follows:

$$
\begin{gathered}
p_{A x}^{\prime}=\beta \gamma E_{A}^{*} / c+\gamma p^{*} \cos \theta^{*}, \\
p_{A y}^{\prime}=p^{*} \sin \theta^{*}
\end{gathered}
$$

where $E_{A}^{*} / c=\sqrt{\left(p^{*}\right)^{2}+\left(m_{A} c\right)^{2}}=E_{A}^{\prime *} / c$. From these equations and the relation $\cos ^{2} \theta^{*}+\sin ^{2} \theta^{*}=1$, we obtain

$$
\left(\frac{p_{A x}^{\prime}-\beta \gamma E_{A}^{*} / c}{\gamma p^{*}}\right)^{2}+\left(\frac{p_{A y}^{\prime}}{p^{*}}\right)^{2}=1 .
$$

This equation indicates the ellipse [4] whose parameters

$$
\begin{gathered}
\text { minor semiaxis } p^{*}=\frac{p_{A} m_{B} c}{\sqrt{\left(m_{A} c\right)^{2}+\left(m_{B} c\right)^{2}+2 E_{A} m_{B}}}, \\
\text { major semiaxis } \gamma p^{*}=\beta \gamma E_{B}^{*} / c=\frac{p_{A}\left\{\left(m_{B} c\right)^{2}+E_{A} m_{B}\right\}}{\left(m_{A} c\right)^{2}+\left(m_{B} c\right)^{2}+2 E_{A} m_{B}}, \\
\text { eccentricity } \beta \gamma p^{*}=\frac{p_{A}^{2} m_{B} c}{\left(m_{A} c\right)^{2}+\left(m_{B} c\right)^{2}+2 E_{A} m_{B}}, \\
\text { midpoint of foci } \beta \gamma E_{A}^{*} / c=\frac{p_{A}\left\{\left(m_{A} c\right)^{2}+E_{A} m_{B}\right\}}{\left(m_{A} c\right)^{2}+\left(m_{B} c\right)^{2}+2 E_{A} m_{B}},
\end{gathered}
$$

are uniquely determined by the initial conditions of the collision. The energy of the target in the center-of-mass system is defined by $E_{B}^{*} / c=\sqrt{\left(p^{*}\right)^{2}+\left(m_{B} c\right)^{2}}=E_{B}^{* *} / c$, which is the same in magnitude before and after the collision.

\section{Diagrammatic Technique}

In this section, we deduce all relations, which we recalled in the former section, from the diagrammatic technique.

Firstly, we draw a dashed circle whose radius is $p_{A}^{*}=p_{B}^{*}=p^{*}$ in Equation (3), as depicted in Figure 2. The dashed circle shows the collision in the 


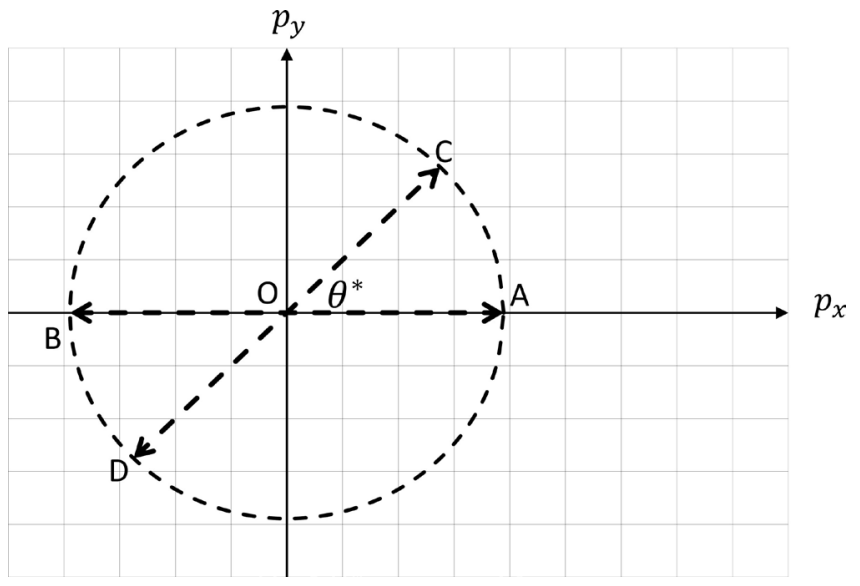

Figure 2. Collision in the center-of-mass system. The figure shows that the masses $m_{A} c=4$ and $m_{B} c=6$ and the velocity $v_{A}=0.9 c$ denote $p^{*}=3.892$. The vectors $\boldsymbol{O A}=\boldsymbol{p}_{A}^{*}$ and $\boldsymbol{O B}=\boldsymbol{p}_{B}^{*}$ are the momenta of incident particles before the collision. The vectors $\boldsymbol{O C}=\boldsymbol{p}_{A}^{\prime *}$ and $\boldsymbol{O D}=\boldsymbol{p}_{B}^{\prime *}$ are the momenta of outgoing particles after the collision. The angle $\angle C O A=\theta^{*}$ is not determined by the conservation laws only. We fix it according to the given collision problems.

center-of-mass system. Now, we draw arrows of momenta into the circle. The momenta before the collision are supposed to be along the $x$-axis

$$
\boldsymbol{O A}=\boldsymbol{p}_{A}^{*}, \quad \boldsymbol{O B}=\boldsymbol{p}_{B}^{*}=-\boldsymbol{p}_{A}^{*} .
$$

After the collision, the momenta stay the same in magnitude, but change the direction

$$
\boldsymbol{O C}=\boldsymbol{p}_{A}^{\prime *}, \quad O D=\boldsymbol{p}_{B}^{\prime *}=-\boldsymbol{p}_{A}^{\prime *} .
$$

Since the scattering angle $\theta^{*}=\angle C O A$ cannot be determined by the conservations of momentum and energy, the point $C$ lies anywhere on the circle and the point $D$ is opposite side against the point $C$. It is determined according to what we are asked in the collision problems.

Next, as shown in Figure 3, we draw an ellipse according to Equation (6) with the parameters from Equations (7) to (10). The point $E$ is the midpoint of the foci $E^{\prime}$ and $E^{\prime}$. This ellipse signifies $O E=\beta \gamma E_{A}^{*} / c$ and $E G=\beta \gamma E_{B}^{*} / c$, and we find from Equations (8) and (10) that

$$
O G=p_{A}=\beta \gamma E_{A}^{*} / c+\beta \gamma E_{B}^{*} / c=O E+E G
$$

is the momentum of the projectile in the laboratory system before the collision.

Next, as depicted in Figure 4, we draw a broken line from the point $C$ in parallel to the $p_{x}$-axis until the broken line intersects with the ellipse. We call this point of intersection as $F$. Then, the vector $\boldsymbol{O F}=\boldsymbol{p}_{A}^{\prime}$ becomes the momentum of the projectile $A$ after the collision. The angle $\angle F O G=\theta$ is the scattered angle of the particle $A$ in the laboratory system. We note that the angel $\theta^{*}$ in Figure 2 and the angle $\theta$ in Figure 4 are related each other. Once the $\theta^{*}$ is fixed by the given collision problems, the $\theta$ is determined according to the prescription stated above. And the converse is also true. If the collision problem 


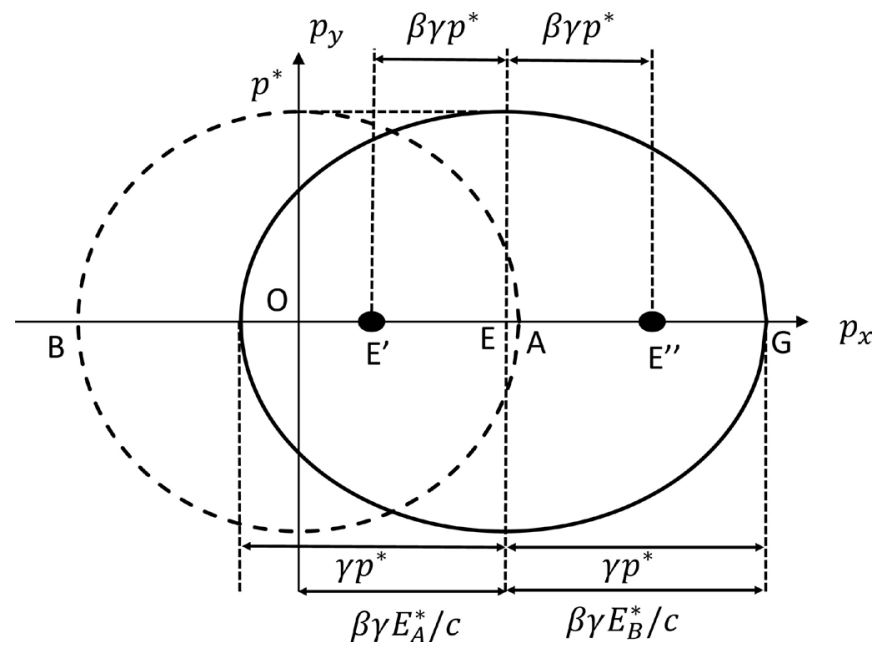

Figure 3. The ellipse Equation (6) can be drawn according as the parameters from Equations (7) to (10). The solid circle E' and E" on the $p_{x}$-axis are the foci of this ellipse. The point $E$ is the midpoint of the foci.

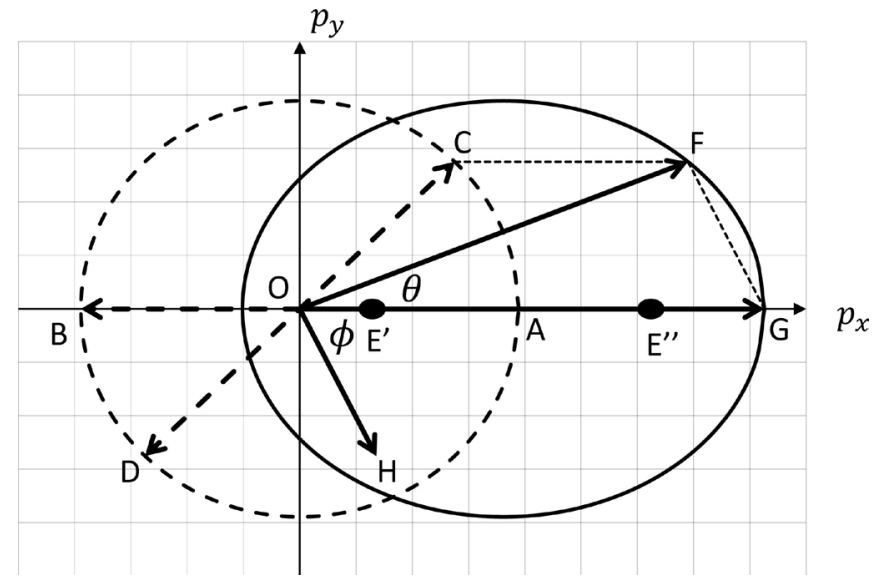

Figure 4. The ellipse implies the collision in the laboratory system in case of the incident velocity $v_{A}=0.9 c$. The initial condition is as the same with Figure 2 . The solid circles on the $p_{x}$-axis denote the foci of the ellipse whose coordinates are $p_{x}=6.144$ and 1.096. And the eccentricity is 2.524. The vectors $\boldsymbol{O F}=\boldsymbol{p}_{A}^{\prime}$ and $\boldsymbol{O H}=\boldsymbol{F} \boldsymbol{G}=\boldsymbol{p}_{B}^{\prime}$ show the momenta of the projectile and the target after the collision.

gives the angle $\theta$ in the laboratory system, we first draw the vector $\boldsymbol{O F}=\boldsymbol{p}_{A}^{\prime}$ in the ellipse. Then, we trace from $F$ to $C$ along the broken line. The vector $\boldsymbol{O C}=\boldsymbol{p}_{A}^{\prime *}$ shows the momentum of the projectile $A$ in the center-of-mass system. And the angle $\angle C O A=\theta^{*}$ is the scattered angle of this system.

Next, the vector $\boldsymbol{F} \boldsymbol{G}=\boldsymbol{O H}=\boldsymbol{p}_{B}^{\prime}$ shows the momentum of the target $B$ in the laboratory system after the collision. The angle $\angle F G O=\angle G O H=\phi$ is the scattered angle of the target $B$. The vector $\boldsymbol{O G}=\boldsymbol{O} \boldsymbol{E}+\boldsymbol{E} \boldsymbol{G}=\boldsymbol{O F}+\boldsymbol{O H}$ shows the momentum conservation law $\boldsymbol{p}_{A}=\boldsymbol{p}_{A}^{\prime}+\boldsymbol{p}_{B}^{\prime}$ of the collision.

The ellipse has or has not intersections with $p_{y}$-axis, according as $m_{A}<m_{B}$ or $m_{A}>m_{B}$. It is found from the magnitude of $\gamma p^{*}$ and $\beta \gamma E_{A}^{*} / c$ in Equations (8) and (10). The corresponding diagrams are shown in Figure 4 and 
Figure 5. As we see from Figure 4 that if $m_{A}<m_{B}$, the projectile $A$ can have any direction after the collision. However, in case of $m_{A}>m_{B}$ in Figure 5 , the projectile $A$ can be deflected only through an angle not exceeding $\theta_{\max }$ from its original direction. This case is shown in Figure 6. The maximum value of $\theta_{\max }$ is determined by the position $F$ at which $O F$ is a tangent to the ellipse.

\section{Identical Particles and Newtonian Limit}

The case $m_{A}=m_{B}=m$ becomes quite simple as shown in Figure 7. The parameters of the ellipse are as follows:

$$
\text { minor semiaxis } p^{*}=\frac{m c p_{A}}{\sqrt{2 m c\left(E_{A} / c+m c\right)}} \text {, }
$$

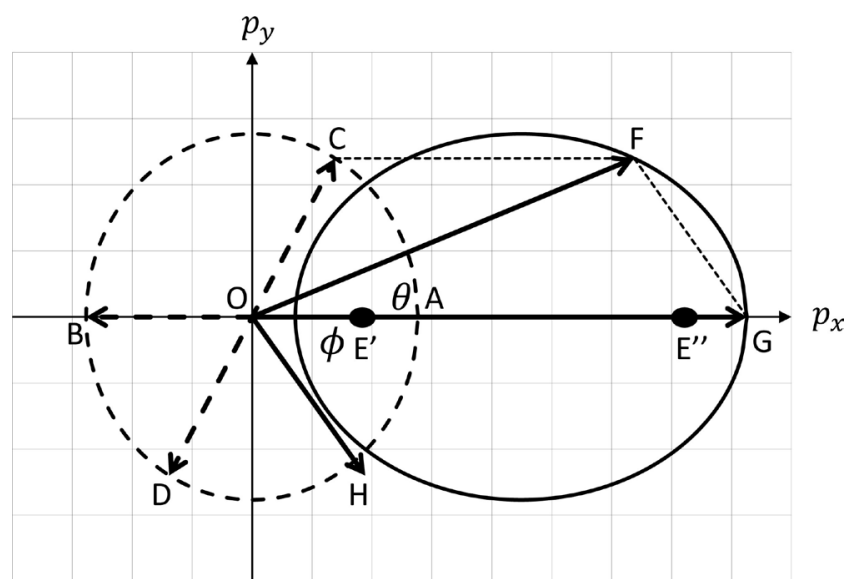

Figure 5. If $m_{A}>m_{B}$, the ellipse does not have intersections with the $p_{y}$-axis. The figure shows that the masses $m_{A} c=4$ and $m_{B} c=3$ and the velocity $v_{A}=0.9 c$ denote $p^{*}=2.769$. The solid circles on the $p_{x}$-axis denote the foci of the ellipse whose coordinates are $p_{x}=7.047$ and 1.935. And the eccentricity is 2.556 .

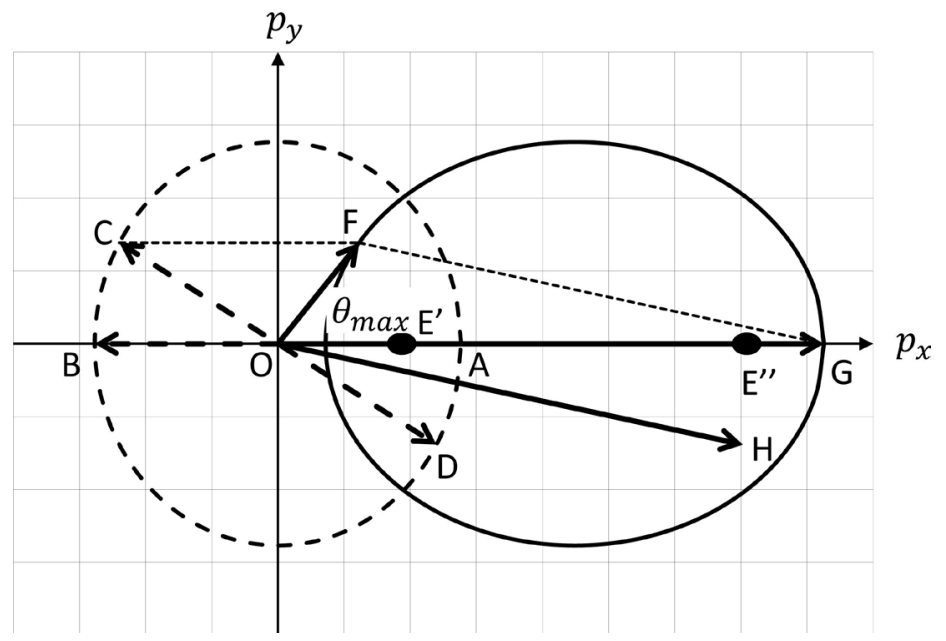

Figure 6. The initial condition is as the same with Figure 5. If $m_{A}>m_{B}$, the projectile $A$ can be deflected only through an angle not exceeding $\theta_{\max }=\angle F O G$ from its original direction. The line segment $O F$ is a tangent of the ellipse. 


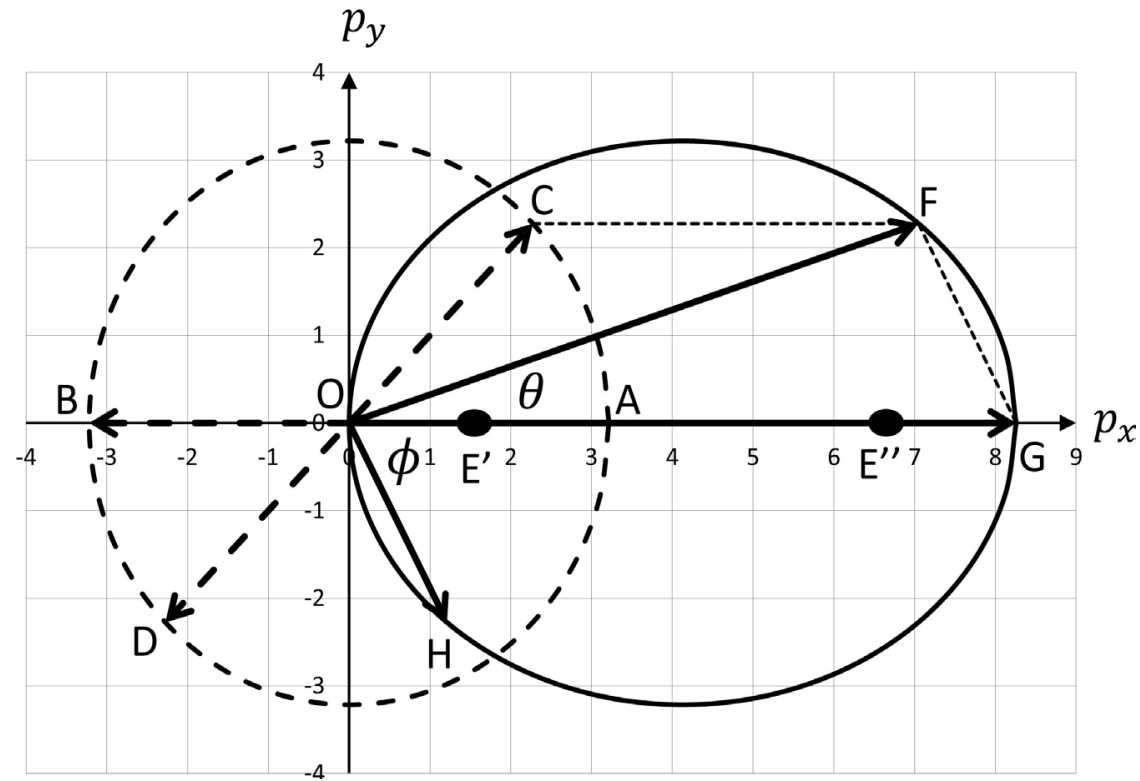

Figure 7. The incident particles have the same mass $m_{A} c=m_{B} c=m c=4$. The $p_{y}$-axis is a tangent of the ellipse. The projectile with velocity $v_{A}=0.9 c$ collides with the target which is at rest. The solid circles on the $p_{x}$-axis denote the foci of the ellipse whose coordinates are $p_{x}=1.541$ and 6.718. And the eccentricity is 2.588 .

$$
\begin{aligned}
& \text { major semiaxis } \gamma p^{*}=\beta \gamma E_{B}^{*} / c=\frac{p_{A}}{2}, \\
& \text { eccentricity } \beta \gamma p^{*}=\frac{E_{A} / c-m c}{2}, \\
& \text { midpoint of foci } \beta \gamma E_{A}^{*} / c=\frac{p_{A}}{2} .
\end{aligned}
$$

In this case, since $\gamma p^{*}=\beta \gamma E_{A}^{*} / c$, the $p_{y}$-axis becomes a tangent to the ellipse and the tip of the vector $\boldsymbol{O H}=\boldsymbol{p}_{B}^{\prime}$ is also on the ellipse.

The cases of which the initial speed of the projectile $A$ has $v_{A}=0.6 c$ and $v_{A}=0.1 c$ are shown in Figure 8 and Figure 9. Here, we note that as we see from Equations (14)-(17), the different speed of the incident particle gives the different ellipse in magnitude. As the speed of the incident particle decreases, the eccentricity of the ellipse decreases and the ellipse begins to resemble a circle [3] [5]. It is found that if we take the limit with $c \rightarrow \infty$, the parameters in Equations (7)-(10) become

$$
\begin{gathered}
\text { minor semiaxis } p^{*} \rightarrow \frac{m_{B}}{m_{A}+m_{B}} p_{A}, \\
\text { major semiaxis } \gamma p^{*}=\beta \gamma E_{B}^{*} / c \rightarrow \frac{m_{B}}{m_{A}+m_{B}} p_{A}, \\
\text { eccentricity } \beta \gamma p^{*} \rightarrow 0, \\
\text { midpoint of foci } \beta \gamma E_{A}^{*} / c \rightarrow \frac{m_{A}}{m_{A}+m_{B}} p_{A} .
\end{gathered}
$$




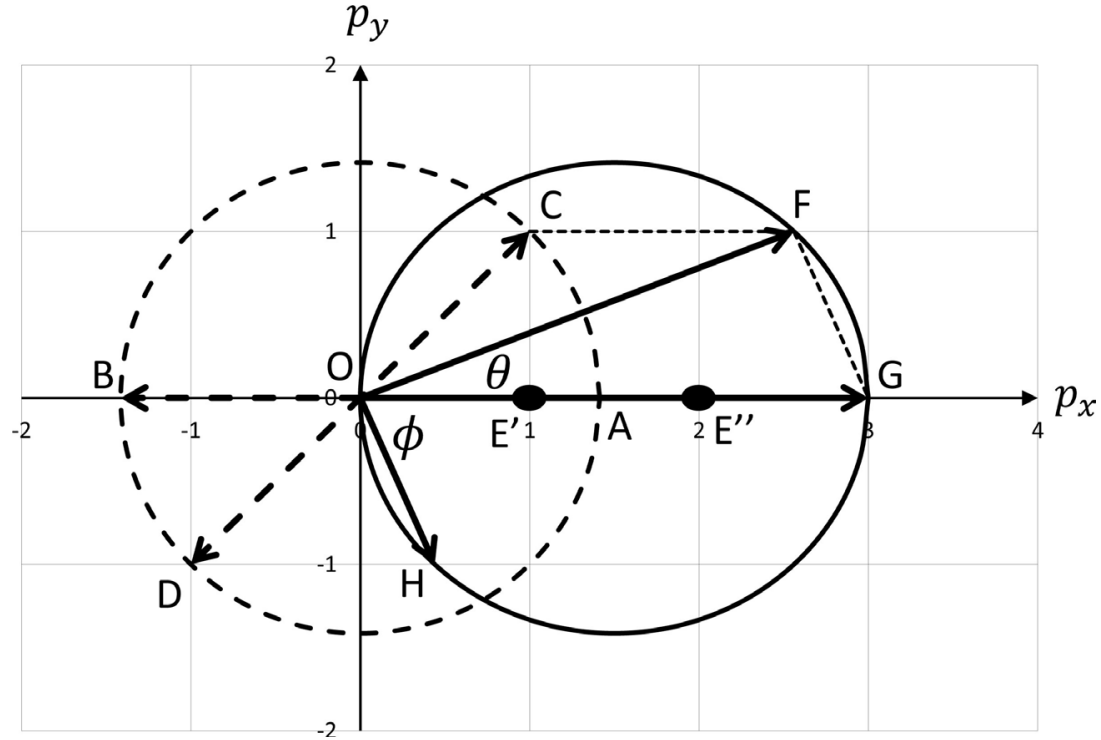

Figure 8. The incident particles have the same mass $m_{A} c=m_{B} c=4$. The projectile $A$ has the velocity $v_{A}=0.6 c$ and the target is at rest. The solid circles on the $p_{x}$-axis denote the foci of the ellipse whose coordinates are $p_{x}=1$ and 2. And the eccentricity is 0.5 .

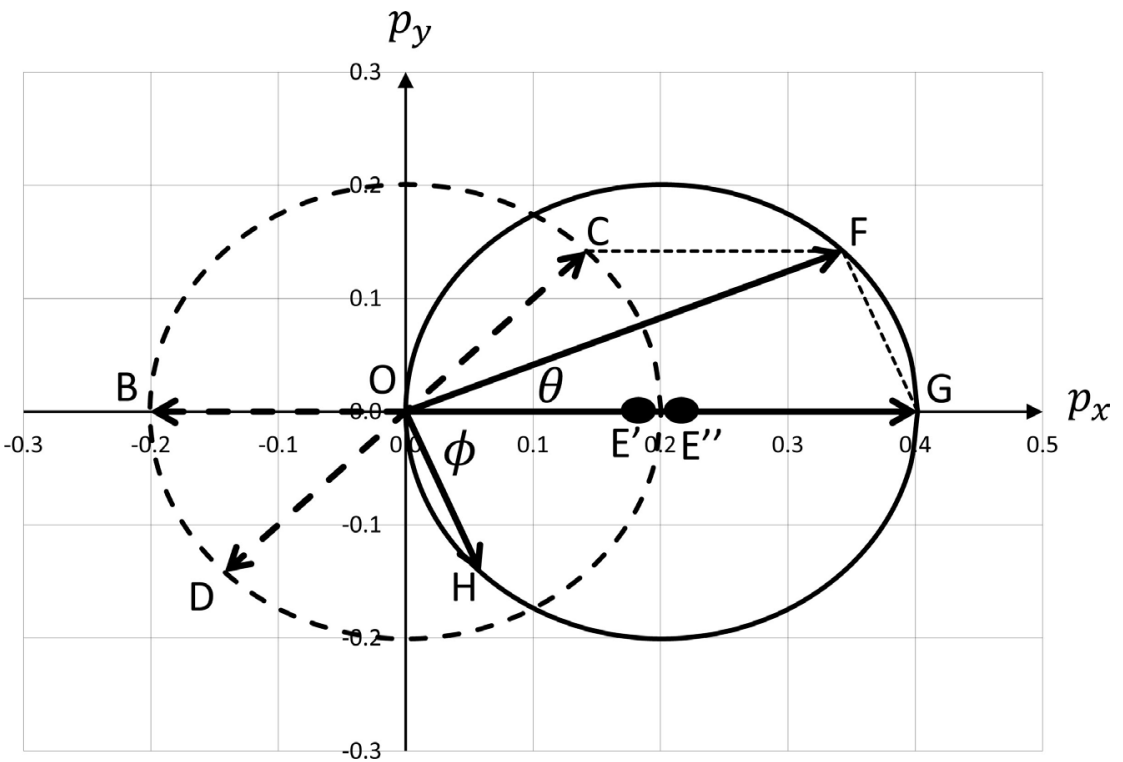

Figure 9. The incident particles have the same mass $m_{A} c=m_{B} c=4$. The projectile $A$ has the velocity $v_{A}=0.1 c$ and the target is at rest. The solid circles on the $p_{x}$-axis denote the foci of the ellipse whose coordinates are $p_{x}=0.191$ and 0.211 . And the eccentricity is 0.010 .

The semiaxes become the same length and the eccentricity tends to zero. The case of the Newtonian collision problems [3] [5] is recovered in this limit.

\section{Conclusion}

We derive the diagrammatic presentation of the two dimensional elastic collision problem in the special relativity. We draw the circle for the center-of-mass 
system and the ellipse for the laboratory system. Those circle and ellipse show the whole story of the two dimensional elastic collisions. When we use the graph paper for drawing those figures, we are able to measure the length of momentum vectors and the scattered angle by using the ruler and the protractor. This diagrammatic technique can help us understand collision problems qualitatively and quantitatively.

\section{Acknowledgements}

The author thanks the anonymous reviewer for his helpful suggestions.

\section{Conflicts of Interest}

The authors declare no conflicts of interest regarding the publication of this paper.

\section{References}

[1] Landau, L.D. and Lifshitz, E.M. (1976) Mechanics. Butterworth-Heinenann, Oxford.

[2] Ogura, A. (2017) Analyzing Collisions in Classical Mechanics Using Mass-Momentum Diagrams. European Journal of Physics, 38, 055001. https://doi.org/10.1088/1361-6404/aa750b

[3] Ogura A. (2018) Diagrammatic Approach for Investigating Two Dimensional Elastic Collisions in Momentum Space I: Newtonian Mechanics. World Journal of Mechanics, 8.

[4] Landau, L.D. and Lifshitz, E.M. (1976) The Classical Theory of Fields. Butterworth-Heinenann, Oxford.

[5] Bokor, N. (2011) Analysing Collisions Using Minkowski Diagrams in Momentum Space. European Journal of Physics, 32, 773. https://doi.org/10.1088/0143-0807/32/3/013

[6] Goldstein, H., Poole, C. and Safko, J. (2002) Classical MECHANICS. Addison Wesley, Boston. 